\title{
Prediction of cancer cell sensitivity to natural products based on genomic and chemical properties
}

Zhenyu Yue, Wenna Zhang, Yongming Lu, Qiaoyue Yang, Qiuying Ding, Junfeng Xia, Yan Chen

Natural products play a significant role in cancer chemotherapy. They are likely to provide many lead structures, which can be used as templates for the construction of novel drugs with enhanced antitumor activity. Traditional research approaches studied structureactivity relationship of natural products and obtained key structural properties, such as a type of chemical bond or group, with the purpose of ascertaining their effect on a single cell line or a single tissue type. Here, for the first time, we develop a machine learning method to comprehensively predict natural products responses against a panel of cancer cell lines based on both the gene expression and the chemical properties of natural products. The results on two datasets, training set and independent test set, show that this proposed method yields significantly better prediction accuracy. In addition, we also demonstrate the predictive power of our proposed method by modeling the cancer cell sensitivity to two natural products, Curcumin and Resveratrol, which indicate that our method can effectively predict the response of cancer cell lines to these two natural products. Taken together, the method will facilitate the identification of natural products as cancer therapies and the development of precision medicine by linking the features of patient genomes to natural product sensitivity. 
$1 \quad$ Prediction of cancer cell sensitivity to natural products

4 Zhenyu Yue ${ }^{1}$, Wenna Zhang ${ }^{1}$, Yongming Lu ${ }^{1}$, Qiaoyue Yang ${ }^{1}$, Qiuying Ding ${ }^{1}$,

5 Junfeng $\mathrm{Xia}^{2, *}$ and Yan Chen ${ }^{1, *}$

6 'School of Life Sciences, Anhui University, Hefei, Anhui 230601, China

7 '2Institute of Health Sciences, School of Computer Science and Technology, Anhui

8 University, Hefei, Anhui 230601, China

9

$10{ }^{*}$ Corresponding author:

11 Tel: +86-551-63861751; E-mail: chenyan91030@yahoo.com (Yan Chen)

12 Tel: +86-551- 63861990; E-mail: jfxia@ahu.edu.cn (Junfeng Xia)

13

14

15

16

17

18

19

20 
Abstract

Natural products play a significant role in cancer chemotherapy. They are likely to provide many lead structures, which can be used as templates for the construction of novel drugs with enhanced antitumor activity. Traditional research approaches studied structure-activity relationship of natural products and obtained key structural properties, such as a type of chemical bond or group, with the purpose of ascertaining their effect on a single cell line or a single tissue type. Here, for the first time, we develop a machine learning method to comprehensively predict natural products responses against a panel of cancer cell lines based on both the gene expression and the chemical properties of natural products. The results on two datasets, training set and test set, show that the proposed method yields significantly better prediction accuracy. In addition, we also demonstrate the predictive power of our proposed method by modeling the cancer cell sensitivity to two natural products, Curcumin and Resveratrol, which indicate that our method can effectively predict the response of cancer cell lines to these two natural products. Taken together, the method will facilitate the identification of natural products as cancer therapies and the development of precision medicine by linking the features of patient genomes to natural product sensitivity. 
43

44

45

46

47

48

\section{Introduction}

In recent years, many natural products were purified and shown to have cancer chemopreventive activity in laboratory, as exemplified by Camptothecin, Vinblastine, Embelin and Paclitaxel (Dai et al., 2011; Goldwasser et al., 1995; Lynch et al., 2012; of Trialists, 2011). These agents from natural source have contributed significantly to the successful treatment of melanoma, leukemia, breast cancer and many other carcinomas. In addition, more and more new derivatives based on the structure of natural products have become promising candidates for antitumor drugs through laboratory design, synthesis and screening (Chen et al., 2006; Rodríguez-Berna et al., 2014; Silvestri, 2013). However, experimental methods for searching natural product lead structure suffered from the drawbacks of expensive and time-consuming. Therefore the use of computational methods based on structure-activity relationship (SAR) has been intensively investigated.

Traditional exploring approaches of SAR focus on producing a range of analogues based on the basic skeleton of lead structure by synthetic chemists and searching empirically for their structural properties predictive of the antitumor activity (Cao et al., 2013; Dong et al., 2012; Liu et al., 2015; Zhang et al., 2007). These structure-activity relationship studies tried to predict responses in a single cell line or a single tissue type using only structure data. Although much progress has been made, the problem of predicting natural products response is far from being solved. 
response to natural products, based on gene expression of cancer cell lines (genomic information) and the chemical descriptors of the considered natural products (chemical structure) for the first time. Empirical studies show that our method can obtain good performance when predicting sensitivity for hundreds of cancer cell lines to natural products in test set and case study analyses and indicate that both the structural properties and gene expression signatures are important determinants of antitumor activity of natural products. Taken together, this study outlines a first approach to predict drug response for natural products and generate novel natural product candidates for further studies.

\section{Materials and Methods}

\subsection{Data collection}

In order to develop robust predictors of response to natural products, we collected and annotated a published large-scale preclinical dataset, namely, the Genomics of Drug Sensitivity in Cancer (GDSC) (Garnett et al., 2012). This large dataset includes drug sensitivity data from 138 drugs across almost 700 cell lines. By retrieving the drug information from PubChem database (http://pubchem.ncbi.nlm.nih.gov), we identified 17 drugs as natural products or their derivatives from these 138 drugs (Table 1). These natural products in GDSC were screened across a range of $\mathbf{2 7 9 - 5 6 5}$ cell lines per drug (mean $=\mathbf{4 9 5}$ cell lines per drug) representing $\mathbf{8 4 2 0}$ cancer cell line-drug interactions. The publically available drug sensitivity (Drug $\mathrm{IC}_{50}$ values) data for all the 17 natural products was downloaded from GDSC (http://www.cancerrxgene.org). Among these 17 natural products, 13 of them were randomly chosen for models' building, which represents 6450 cancer cell line-natural product interactions (training set, Table S1). The 
84

85

86

87

rest 4 natural products were used in the test set containing 1970 cancer cell line-natural product interactions (Table S2).

An independent test set (case studies) was extracted from the literature to further assess the performance of our proposed method. By searching anticancer herbs database of systems pharmacology (CancerHSP) (Tao et al., 2015) and natural products-related studies from the PubMed (http://www.ncbi.nih.gov/pubmed), we obtained two antitumor natural products (Curcumin and Resveratrol), which have been proven effective in inhibiting proliferation and inducing apoptosis of various kinds of cancer cell lines. For Curcumin, it was screened on 16 cancer cell lines derived from 5 cancer types; and for Resveratrol, it encompasses drug sensitivity data for 13 cancer cell lines derived from 6 cancer types. After removing cell lines for which we could not find the corresponding gene expression information in GDSC, we finally obtained 7 and 8 cancer cell line-natural product interactions for Curcumin and Resveratrol, respectively (Table 2).

\subsection{Genomic features}

The GDSC gene expression microarray data were derived directly from the work of Geeleher et al. (Geeleher et al., 2014). Subsequent analyses were restricted to the gene with Entrez gene ID, for a total of 12026 genes.

\subsection{Chemical features}

Natural products chemical features were generated with PaDEL software (Yap, 2011) from the simplified molecular-input line entry system (SMILES) (Weininger, 1988). The SMILES files for natural products were collected manually from PubChem database 
105 (http://pubchem.ncbi.nlm.nih.gov). Initially, we obtained 1444 1-D and 2-D descriptors of

106

107

108

109

110

111

112

113

114

115

116

117

118

119

120

121

natural products directly from PaDEL. The chemical features with the same value across all

natural products were further eliminated. Finally, we obtained 1114 chemical features in this

study.

\subsection{Models construction}

In this study, the prediction model was built using the software WEKA (Hall et al., 2009) with the default parameters. The R scripts (Ihaka and Gentleman, 1996) were used for the statistical analyses.

\section{Results and Discussion}

\subsection{Strategy for prediction of cancer cell sensitivity to natural products}

Our goal was to use gene expression and in vitro drug sensitivity data derived from cell lines, with the addition of chemical properties, to predict cell lines' response to natural products. The conceptual framework for prediction of cancer cell sensitivity to natural products is shown in Figure 1. In the first step, cell lines in GDSC were clustered into two groups (Sensitive and Resistant) or three groups (Sensitive, Resistant and Intermediate) according to their sensitivities (drug $\mathrm{IC}_{50}$ values) to a given drug with K-Means algorithm in WEKA (Hall et al., 2009). Here K was set 2 or 3 , which means that the cancer cell lines were divided into 2 or 3 groups. Samples in Sensitive and Resistant groups are used to build machine learning model. Here, the performance of J48 (Decision Tree), SVM (Support Vector Machine), Random Forest and Rotation Forest (Rodriguez et al., 2006) models were comprehensively evaluated. After this step, we used genomic features from gene expression data and chemical features to construct prediction model, 
126 127 2011).

128

129

130

131

132

133

134

135

136

137

138

139

140

141

142

143

144

145

146

where the optimal feature number were selected using t-test with R scripts (Gentleman et al.,

\subsection{Determination of number of cancer cell lines clusters}

To find the optimal number of cancer cell lines clusters in K-Means algorithm, the prediction performance of different clusters $(\mathrm{K})$ were evaluated based on 10-fold cross validation (training set) and test set using Rotation Forest models. As can be seen in Figure 2, the AUC (Area under the receiver operating characteristic curve) is higher in the case $K=3$ compared with those in the case $\mathrm{K}=2$ when features number is set as 50 . The similar situation occurred when the features number is set as 100 or 500 (Figure S1 and Figure S2, respectively), so we chose $\mathrm{K}=3$, which means that the cancer cell lines in GDSC were clustered into three groups (Sensitive, Resistant and Intermediate), and only cell lines in Sensitive and Resistant groups were used in the subsequent analyses.

\subsection{Assessment of feature importance}

In feature selection step, a 10-fold cross validation on the training set was conducted to get the optimal gene numbers. Examination on predicted AUC with respect to numbers of selected feature numbers showed a consistent trend of increasing first and decreasing afterwards with the increase of selected feature numbers except SVM model (Figure 3). As a result, the top 1000 features were chosen as optimal features for further analyses.

There were 468 genes (genomic features) in the top 1000 features, of which 59 genes are cancer related genes (oncogenes or tumor suppressor genes), where oncogenes were obtained from database Cancer Gene Census (Futreal et al., 2004), and tumor suppressor genes were from 
147 database TSGene (Zhao et al., 2013). We carried out a permutation test as follows. We randomly

148 sampled 468 genes from the whole 12026 genes 1000 times, and the mean of the number of

149 overlapped genes was only 36.2. In addition, the maximum value in the 1000 times tests was 54 ,

150 which is also less than 59. A $P$-value zero was obtained in this permutation test. So the genomic

151 features (genes) we chosen were more likely to be related to tumorigenesis.

152 The top 1000 features also contained 532 chemical descriptors of natural products. The

153 systematic machine learning-based integration of various data sources, including chemical

154 structure and genomic information, can provide better discriminative power than those using

155 only individual data sources. This may presents a simple and promising strategy to predict

antitumor activity of unknown natural products using pharmacology data and machine learning

approaches.

158 The detailed 468 genes and 532 descriptors used in the top 1000 features are shown in

Supplementary material Table S3 and Table S4.

160

161

162

163

164

165

166

167

\subsection{Comparison of different machine learning methods}

In this study, in order to identify the best machine learning technique suitable for predicting cancer cell sensitivity to natural products, we comprehensively evaluated the performances of SVM (LibSVM), Decision Tree (J48), Random Forest, and Rotation Forest classifiers. All these algorithms were implemented using the Weka package with the default parameter configuration. Rotation Forest has been proven to be a relatively stable machine learning method in our previous work (Xia et al., 2010), which also performed best using 10-fold cross validation (AUC $=0.87$, Figure 3 ) in this study. A consistent trend occurred in the test set (Figure 4), where the 
168

169

170

171

172

173

174

175

176

177

178

179

180

181

182

183

184

185

186

187

188

AUC for for Camptothecin, Epothilone B, Paclitaxel, and Shikonin are 0.88, 0.89, 0.79 and 0.81, respectively.

The detailed classifiers assessment results of 10-fold cross validation (training set) and test set are shown in Table 3. The number of cancer cell line-natural product interactions for Camptothecin, Epothilone B, Paclitaxel and Shikonin are 321, 303, 168 and 244, respectively. The performance of each model is measured by five metrics: Precision, Recall, F-Measure, AUC and Accuracy (Fawcett, 2006), where Precision, Recall and F-Measure are calculated for each class, AUC and Accuracy are automatically weighted in WEKA for all classes. As is shown in Table 3, all the 4 methods obtained good results based on 10-fold cross validation (training set) and test data set.

\subsection{Case studies}

To further illustrate the effectiveness of our approach for detecting cancer cell sensitivity to natural products, we present two additional natural products examples. By searching CancerHSP database (Tao et al., 2015) and natural products-related studies from the PubMed database (http://www.ncbi.nih.gov/pubmed), we obtained 2 natural products screened on 29 cancer cell lines: Curcumin (Bush et al., 2001; Choudhuri et al., 2002; Khor et al., 2006; Radhakrishna Pillai et al., 2004; Wang et al., 2006) and Resveratrol (Chen et al., 2004; Clément et al., 1998; Ding and Adrian, 2002; Hsieh and Wu, 1999; Lu and Serrero, 1999; Niles et al., 2003; Whyte et al., 2007), which have been proven effective in prevention and treatment of various kinds of cancers, including melanoma, lung cancer, ovarian cancer and so on (Tao et al., 2015). After eliminating cancer cell lines for which we could not find the corresponding gene expression information in 
189

190

191

192

193

194

195

196

197

198

200

201

202

203

204

205

206

207

208

209

GDSC, we finally obtained 7 and 8 cancer cell line-natural product interactions for Curcumin

and Resveratrol, respectively. The prediction results in these two natural products are shown in

\section{Table 2.}

Case study 1: Curcumin. Curcumin, a phenolic compound from the rhizome of the plant Curcuma longa, induced apoptosis in tumor cells via a p53-dependent pathway or pathways independent of $\mathrm{p} 53$. We predicted responses of 7 cell lines that are sensitive to Curcumin, including 4 cell lines from melanoma, 1 cell line from lung cancer, 1 cell line from breast cancer, and 1 cell line from pancreatic cancer (Table S5). Notably, of the 7 cell lines that were defined as responders, 6 were correctly classified by our model (Table 2). The only cell line that was classified incorrectly is Sk-mel-5, a melanoma cell line containing wild-type p53. Because the rest 3 melanoma cell lines in this study contain mutant p53 (Bush et al., 2001), this may explain why our method could not obtain the correct result in Sk-mel-5 cell line.

Case study 2: Resveratrol. Resveratrol, a plant polyphenol found in grapes and a variety of human foods, is reported to have protective effects against various cancers. The mechanisms of its action in these diseases are inducing apoptosis via different pathways, antiestrogenic effect and so on. Responses of 8 cell lines to Resveratrol were predicted in this study, including 2 cell lines from melanoma, 1 cell line from lung cancer, 3 cell line from breast cancer, 1 cell line from pancreatic cancer and 1 cell line from prostate cancer (Table S5). SK-MEL-28, one of the two human melanoma cell lines used here, was predicted to be sensitive. The other melanoma cell line, A375, is amelanotic differing from the former. And Resveratrol induced phosphorylation of ERK1/2 in A375 which can promote gene expression associated with proliferation and 
210 differentiation, but not in SK-mel28 cells. Whether these differences contribute to the incorrect

211 prediction of A375 cell line response to Resveratrol remains to be determined. Breast and

212 prostate cell lines used here were all classified correctly. Altogether, 5 out of the 8 cancer cell

213 line-natural product interactions can be correctly predicted by our model (Table 2).

\section{4. Conclusions}

215 In this study, we investigated the inherent determinants of antitumor activity of natural

products. For this purpose, we developed a machine learning method to predict natural products

responses against a panel of cancer cell lines based on both the gene expression data and the

chemical properties of natural products. Our results show that it is possible to enrich for natural

products responders using gene expression and chemical descriptors, by applying models

generated from a large panel of cancer cell lines. The performance of our approach was firstly

evaluated using the 10-fold cross validation (training set) and test set, and further validated by

modeling two additional natural products (case studies analyses). The experimental results show

that our method can effectively predict the response of cancer cell lines to natural products.

Although our final best model is based on both the gene expression signatures of cancer cells

lines and the chemical properties, novel features that better describe natural product sensitivity

can be easily incorporated into our prediction system to further improve the prediction

performance of natural product response. In our future work, we will add other genomic features

such as mutation information into the prediction model. Besides these genomic information,

epigenetic and protein level information also play very important role in natural product response 
231 noted that in the current study we focused on "natural product sensitivity in cancer". In the future,

232 we will consider extending our model to non-natural product sensitivity prediction. Last, we will

233 offer an online web interface through which our approach can be implemented to

234 computationally predict natural product sensitivity. 
237

238

239

240

241

242

243

244

245

246

247

248

249

250

251

252

253

254

255

256

257

258

259

260

261

262

263

264

265

266

267

268

269

270

271

272

273

274

275

Bush, J.A., Cheung Jr, K., John, J., and Li, G. (2001). Curcumin induces apoptosis in human melanoma cells through a Fas receptor/caspase-8 pathway independent of p53. Experimental cell research 271, 305-314.

Cao, R., Fan, W., Guo, L., Ma, Q., Zhang, G., Li, J., Chen, X., Ren, Z., and Qiu, L. (2013). Synthesis and structureactivity relationships of harmine derivatives as potential antitumor agents. European journal of medicinal chemistry $60,135-143$.

Chen, J., Nikolovska-Coleska, Z., Wang, G., Qiu, S., and Wang, S. (2006). Design, synthesis, and characterization of new embelin derivatives as potent inhibitors of X-linked inhibitor of apoptosis protein. Bioorganic \& medicinal chemistry letters 16, 5805-5808.

Chen, Y., Tseng, S.-H., Lai, H.-S., and Chen, W.-J. (2004). Resveratrol-induced cellular apoptosis and cell cycle arrest in neuroblastoma cells and antitumor effects on neuroblastoma in mice. Surgery 136, 57-66.

Choudhuri, T., Pal, S., Agwarwal, M.L., Das, T., and Sa, G. (2002). Curcumin induces apoptosis in human breast cancer cells through p53-dependent Bax induction. FEBS letters 512, 334-340.

Clément, M.-V., Hirpara, J.L., Chawdhury, S.-H., and Pervaiz, S. (1998). Chemopreventive agent resveratrol, a natural product derived from grapes, triggers CD95 signaling-dependent apoptosis in human tumor cells. Blood 92 , 996-1002.

Dai, Y., DeSano, J., Qu, Y., Tang, W., Meng, Y., Lawrence, T.S., and Xu, L. (2011). Natural IAP inhibitor Embelin enhances therapeutic efficacy of ionizing radiation in prostate cancer. American journal of cancer research $1,128$.

Ding, X.-Z., and Adrian, T.E. (2002). Resveratrol inhibits proliferation and induces apoptosis in human pancreatic cancer cells. Pancreas 25, e71-e76.

Dong, G., Wang, S., Miao, Z., Yao, J., Zhang, Y., Guo, Z., Zhang, W., and Sheng, C. (2012). New tricks for an old natural product: discovery of highly potent evodiamine derivatives as novel antitumor agents by systemic structureactivity relationship analysis and biological evaluations. Journal of medicinal chemistry 55, 7593-7613.

Fawcett, T. (2006). An introduction to ROC analysis. Pattern Recognition Letters 27, 861-874.

Futreal, P.A., Coin, L., Marshall, M., Down, T., Hubbard, T., Wooster, R., Rahman, N., and Stratton, M.R. (2004). A census of human cancer genes. Nature Reviews Cancer 4, 177-183.

Garnett, M.J., Edelman, E.J., Heidorn, S.J., Greenman, C.D., Dastur, A., Lau, K.W., Greninger, P., Thompson, I.R., Luo, X., and Soares, J. (2012). Systematic identification of genomic markers of drug sensitivity in cancer cells. Nature 483, 570-575.

Geeleher, P., Cox, N.J., and Huang, R.S. (2014). Clinical drug response can be predicted using baseline gene expression levels and in vitro drug sensitivity in cell lines. Genome biology 15, R47.

Gentleman, R., Carey, V., Huber, W., and Hahne, F. (2011). Genefilter: Methods for filtering genes from microarray experiments. R package version 1.

Goldwasser, F., Bae, I., Valenti, M., Torres, K., and Pommier, Y. (1995). Topoisomerase I-related parameters and camptothecin activity in the colon carcinoma cell lines from the National Cancer Institute anticancer screen. Cancer research 55, 2116-2121.

Hall, M., Frank, E., Holmes, G., Pfahringer, B., Reutemann, P., and Witten, I.H. (2009). The WEKA data mining software: an update. ACM SIGKDD explorations newsletter 11, 10-18.

Hsieh, T.-c., and Wu, J.M. (1999). Differential effects on growth, cell cycle arrest, and induction of apoptosis by resveratrol in human prostate cancer cell lines. Experimental cell research 249, 109-115. 
Ihaka, R., and Gentleman, R. (1996). R: a language for data analysis and graphics. Journal of computational and graphical statistics 5, 299-314.

Khor, T.O., Keum, Y.-S., Lin, W., Kim, J.-H., Hu, R., Shen, G., Xu, C., Gopalakrishnan, A., Reddy, B., and Zheng, X. (2006). Combined inhibitory effects of curcumin and phenethyl isothiocyanate on the growth of human PC-3 prostate xenografts in immunodeficient mice. Cancer research 66, 613-621.

Liu, T., Ma, M., Ge, H.-M., Yang, C., Cleveland, J., and Shen, B. (2015). Synthesis and evaluation of 8, 4' dideshydroxy-leinamycin revealing new insights into the structure-activity relationship of the anticancer natural product leinamycin. Bioorganic \& Medicinal Chemistry Letters.

Lu, R., and Serrero, G. (1999). Resveratrol, a natural product derived from grape, exhibits antiestrogenic activity and inhibits the growth of human breast cancer cells. Journal of cellular physiology 179, 297-304.

Lynch, T.J., Bondarenko, I., Luft, A., Serwatowski, P., Barlesi, F., Chacko, R., Sebastian, M., Neal, J., Lu, H., and Cuillerot, J.-M. (2012). Ipilimumab in combination with paclitaxel and carboplatin as first-line treatment in stage IIIB/IV non-small-cell lung cancer: Results from a randomized, double-blind, multicenter phase II study. Journal of Clinical Oncology 30, 2046-2054.

Niles, R.M., McFarland, M., Weimer, M.B., Redkar, A., Fu, Y.-M., and Meadows, G.G. (2003). Resveratrol is a potent inducer of apoptosis in human melanoma cells. Cancer Letters 190, 157-163.

of Trialists, I.C. (2011). International phase III trial assessing neoadjuvant cisplatin, methotrexate, and vinblastine chemotherapy for muscle-invasive bladder cancer: long-term results of the BA06 30894 trial. Journal of Clinical Oncology 29, 2171.

Radhakrishna Pillai, G., Srivastava, A.S., Hassanein, T.I., Chauhan, D.P., and Carrier, E. (2004). Induction of apoptosis in human lung cancer cells by curcumin. Cancer letters 208, 163-170.

Rodríguez-Berna, G., Mangas-Sanjuán, V., Gonzalez-Alvarez, M., Gonzalez-Alvarez, I., García-Giménez, J.L., Cabañas, M.J.D., Bermejo, M., and Corma, A. (2014). A promising camptothecin derivative: Semisynthesis, antitumor activity and intestinal permeability. European journal of medicinal chemistry 83, 366-373.

Rodriguez, J.J., Kuncheva, L.I., and Alonso, C.J. (2006). Rotation forest: A new classifier ensemble method. Pattern Analysis and Machine Intelligence, IEEE Transactions on 28, 1619-1630.

Silvestri, R. (2013). New prospects for vinblastine analogues as anticancer agents. Journal of medicinal chemistry 56 , 625-627.

Tao, W., Li, B., Gao, S., Bai, Y., Shar, P.A., Zhang, W., Guo, Z., Sun, K., Fu, Y., and Huang, C. (2015). CancerHSP: anticancer herbs database of systems pharmacology. Scientific reports 5 .

Wang, Z., Zhang, Y., Banerjee, S., Li, Y., and Sarkar, F.H. (2006). Notch - 1 down - regulation by curcumin is associated with the inhibition of cell growth and the induction of apoptosis in pancreatic cancer cells. Cancer 106, 2503-2513.

Weininger, D. (1988). SMILES, a chemical language and information system. 1. Introduction to methodology and encoding rules. Journal of chemical information and computer sciences 28, 31-36.

Whyte, L., Huang, Y.-Y., Torres, K., and Mehta, R.G. (2007). Molecular mechanisms of resveratrol action in lung cancer cells using dual protein and microarray analyses. Cancer research 67, 12007-12017.

Xia, J.-F., Han, K., and Huang, D.-S. (2010). Sequence-based prediction of protein-protein interactions by means of rotation forest and autocorrelation descriptor. Protein and Peptide Letters 17, 137-145.

Yap, C.W. (2011). PaDEL - descriptor: An open source software to calculate molecular descriptors and fingerprints. Journal of computational chemistry 32, 1466-1474. 
317 Zhang, M., Cui, S., Cheung, P., and Wang, Q. (2007). Antitumor polysaccharides from mushrooms: a review on 318 their isolation process, structural characteristics and antitumor activity. Trends in Food Science \& Technology 18, 431919.

320 Zhao, M., Sun, J., and Zhao, Z. (2013). TSGene: a web resource for tumor suppressor genes. Nucleic acids research 321 41, D970-D976. 


\section{Table $\mathbf{1}$ (on next page)}

Natural products and the corresponding cancer cell line-natural product interaction data used in the training set, test set and case studies analyses. 


\begin{tabular}{lcc}
\hline Dataset & Natural product & $\begin{array}{c}\text { Number of cancer cell line-natural product } \\
\text { interaction }\end{array}$ \\
\hline Training set & Vinblastine & 562 \\
& Parthenolide & 281 \\
& Rapamycin & 285 \\
& Thapsigargin & 559 \\
& Bleomycin & 559 \\
& Docetaxel & 562 \\
& Bryostatin 1 & 559 \\
& Cyclopamine & 279 \\
& Cytarabine & 562 \\
& Doxorubicin & 559 \\
& Embelin & 559 \\
& Mitomycin C & 559 \\
& Etoposide & 565 \\
Test set & Camptothecin & 562 \\
& Epothilone B & 559 \\
& Paclitaxel & 284 \\
& Shikonin & 565 \\
Case study & Curcumin & 7 \\
& Resveratrol & 8 \\
\hline
\end{tabular}




\section{Table 2 (on next page)}

Detailed results of case studies.

Samples, the number of cell lines in literature. Overlap, the number of cell lines overlapped with GDSC. Correctly, the number of cell lines whose sensitivity was predicted correctly. 
1 (A) Curcumin

\begin{tabular}{ccccccc}
\hline & Melanoma & Lung & Breast & Pancreas & Prostate & Total \\
\hline Samples & 8 & 2 & 3 & 2 & 1 & 16 \\
Overlap & 4 & 1 & 1 & 1 & 0 & 7 \\
Correctly & 3 & 1 & 1 & 1 & 0 & 6 \\
\hline
\end{tabular}

2 (B) Resveratrol

\begin{tabular}{ccccccccc}
\hline & Melanoma & Lung & $\begin{array}{c}\text { Breas } \\
\mathbf{t}\end{array}$ & Pancreas & $\begin{array}{c}\text { Prostat } \\
\mathbf{e}\end{array}$ & $\begin{array}{c}\text { Neuroblastom } \\
\mathbf{a}\end{array}$ & Total \\
\hline Samples & 2 & 1 & 3 & 2 & 4 & 1 & 13 \\
Overlap & 2 & 1 & 3 & 1 & 1 & 0 & 8 \\
Correctly & 1 & 0 & 3 & 0 & 1 & 0 & 5 \\
\hline
\end{tabular}

3 


\section{Table 3(on next page)}

Results of classification based on different algorithms.

Detailed classifiers assessment results of training set and test set. Each dataset chose the top 1000 group as the model features. J48, Decision Tree. SVM, Support Vector Machines. RF, Random Forest. RoF, Rotation Forest. 
1 (A) Cross Validation

\begin{tabular}{|c|c|c|c|c|c|c|}
\hline Method & Class & Precision & Recall & F-Measure & AUC & Accuracy \\
\hline \multirow[t]{2}{*}{ J48 } & 0 & 0.75 & 0.74 & 0.75 & \multirow{2}{*}{0.75} & \multirow{2}{*}{0.75} \\
\hline & 1 & 0.76 & 0.77 & 0.76 & & \\
\hline \multirow[t]{2}{*}{ SVM } & 0 & 0.77 & 0.78 & 0.77 & \multirow{2}{*}{0.78} & \multirow{2}{*}{0.78} \\
\hline & 1 & 0.79 & 0.77 & 0.78 & & \\
\hline \multirow[t]{2}{*}{ RF } & 0 & 0.75 & 0.83 & 0.79 & \multirow{2}{*}{0.85} & \multirow{2}{*}{0.78} \\
\hline & 1 & 0.82 & 0.74 & 0.78 & & \\
\hline \multirow[t]{2}{*}{ RoF } & 0 & 0.78 & 0.82 & 0.80 & \multirow{2}{*}{0.87} & \multirow{2}{*}{0.80} \\
\hline & 1 & 0.82 & 0.78 & 0.80 & & \\
\hline
\end{tabular}

2

3 (B) Camptothecin

\begin{tabular}{|c|c|c|c|c|c|c|}
\hline Method & Class & Precision & Recall & F-Measure & AUC & Accuracy \\
\hline \multirow[t]{2}{*}{ J48 } & 0 & 0.50 & 0.75 & 0.60 & \multirow{2}{*}{0.61} & \multirow{2}{*}{0.58} \\
\hline & 1 & 0.72 & 0.46 & 0.56 & & \\
\hline \multirow[t]{2}{*}{ SVM } & 0 & 0.80 & 0.63 & 0.70 & \multirow{2}{*}{0.76} & \multirow{2}{*}{0.78} \\
\hline & 1 & 0.77 & 0.90 & 0.82 & & \\
\hline \multirow[t]{2}{*}{ RF } & 0 & 0.73 & 0.76 & 0.75 & \multirow{2}{*}{0.85} & \multirow{2}{*}{0.79} \\
\hline & 1 & 0.82 & 0.80 & 0.81 & & \\
\hline \multirow[t]{2}{*}{ RoF } & 0 & 0.84 & 0.69 & 0.76 & \multirow{2}{*}{0.88} & \multirow{2}{*}{0.82} \\
\hline & 1 & 0.81 & 0.90 & 0.85 & & \\
\hline
\end{tabular}

4

5 (C) Epothilone B

\begin{tabular}{|c|c|c|c|c|c|c|}
\hline Method & Class & Precision & Recall & F-Measure & AUC & Accuracy \\
\hline \multirow[t]{2}{*}{ J48 } & 0 & 0.62 & 0.68 & 0.65 & \multirow{2}{*}{0.75} & \multirow{2}{*}{0.79} \\
\hline & 1 & 0.87 & 0.84 & 0.85 & & \\
\hline \multirow[t]{2}{*}{ SVM } & 0 & 0.34 & 0.99 & 0.51 & \multirow{2}{*}{0.63} & \multirow{2}{*}{0.47} \\
\hline & 1 & 0.98 & 0.26 & 0.41 & & \\
\hline \multirow[t]{2}{*}{ RF } & 0 & 0.58 & 0.92 & 0.71 & \multirow{2}{*}{0.92} & \multirow{2}{*}{0.79} \\
\hline & 1 & 0.96 & 0.74 & 0.83 & & \\
\hline \multirow[t]{2}{*}{ RoF } & 0 & 0.71 & 0.65 & 0.68 & \multirow{2}{*}{0.89} & \multirow{2}{*}{0.83} \\
\hline & 1 & 0.87 & 0.90 & 0.88 & & \\
\hline
\end{tabular}

6

7 (D) Paclitaxel

\begin{tabular}{ccccccc}
\hline Method & Class & Precision & Recall & F-Measure & AUC & Accuracy \\
\hline J48 & 0 & 0.557 & 0.609 & 0.582 & 0.62 & 0.67 \\
& 1 & 0.745 & 0.702 & 0.723 & & \\
SVM & 0 & 0.569 & 0.516 & 0.541 & 0.64 & 0.67 \\
& 1 & 0.718 & 0.76 & 0.738 & & 0.65 \\
\hline
\end{tabular}




\begin{tabular}{|c|c|c|c|c|c|c|}
\hline \multirow{3}{*}{ RoF } & 1 & 0.742 & 0.663 & 0.701 & \multirow{3}{*}{0.79} & \\
\hline & 0 & 0.575 & 0.781 & 0.662 & & \multirow{2}{*}{0.70} \\
\hline & 1 & 0.827 & 0.644 & 0.724 & & \\
\hline
\end{tabular}

8

(E) Shikonin

\begin{tabular}{|c|c|c|c|c|c|c|}
\hline Method & Class & Precision & Recall & F-Measure & AUC & Accuracy \\
\hline \multirow[t]{2}{*}{ J48 } & 0 & 0.71 & 0.59 & 0.65 & \multirow{2}{*}{0.75} & \multirow{2}{*}{0.78} \\
\hline & 1 & 0.81 & 0.88 & 0.84 & & \\
\hline \multirow[t]{2}{*}{ SVM } & 0 & 0.75 & 0.43 & 0.55 & \multirow{2}{*}{0.68} & \multirow{2}{*}{0.76} \\
\hline & 1 & 0.76 & 0.93 & 0.84 & & \\
\hline \multirow[t]{2}{*}{ RF } & 0 & 0.59 & 0.70 & 0.64 & \multirow{2}{*}{0.76} & \multirow{2}{*}{0.73} \\
\hline & 1 & 0.83 & 0.75 & 0.79 & & \\
\hline \multirow[t]{2}{*}{ RoF } & 0 & 0.75 & 0.59 & 0.66 & \multirow{2}{*}{0.81} & \multirow{2}{*}{0.80} \\
\hline & 1 & 0.81 & 0.90 & 0.85 & & \\
\hline
\end{tabular}

9

10 


\section{Figure $\mathbf{1}$ (on next page)}

Natural products sensitivity prediction workflow.

This Data flow diagram demonstrates the simulation workflow for creation and optimization of natural products sensitivity prediction model. Our method was based on three key steps: (1) clustering of IC50 values from the GDSC, then the cell lines were divided into 2 or 3 groups, the sensitive cell lines were set to 1 , the resistant cell lines were set to 0 . (2) Top N features that were most significantly differential between the 1 and 0 cell line sets were chosen as the features of training and test sets. (3) Machine learning models were fitted in WEKA and can then be applied to the new data, to yield natural products sensitivity estimates. 
PeerJ

\section{Ic50 clustering scapt to be reviewed}

\section{Sensitive cell lines I \\ Resistant cell lines 0}

\section{Genes expression levels}

Chemical properties

Top $N$ features

Genes expression levels of cell lines

\section{Chemical properties of new natural products}

New natural products sensitivity 


\section{Figure 2 (on next page)}

Comparison between thecase $\mathrm{K}=2$ and $\mathrm{K}=3$.

Bar chart showing in the case $K=3$ (blue) we obtained a higher AUC than in the case $K=2$ when features number is set as 50 . Cluster3, the case $K=3$. Cluster2, the case $K=2 . C V$, cross validation. Camp, Camptothecin. Epot, Epothilone B. Pacl, Paclitaxel. Shik, Shikonin. AUC, Area under the curve. 


\section{PeerJ}
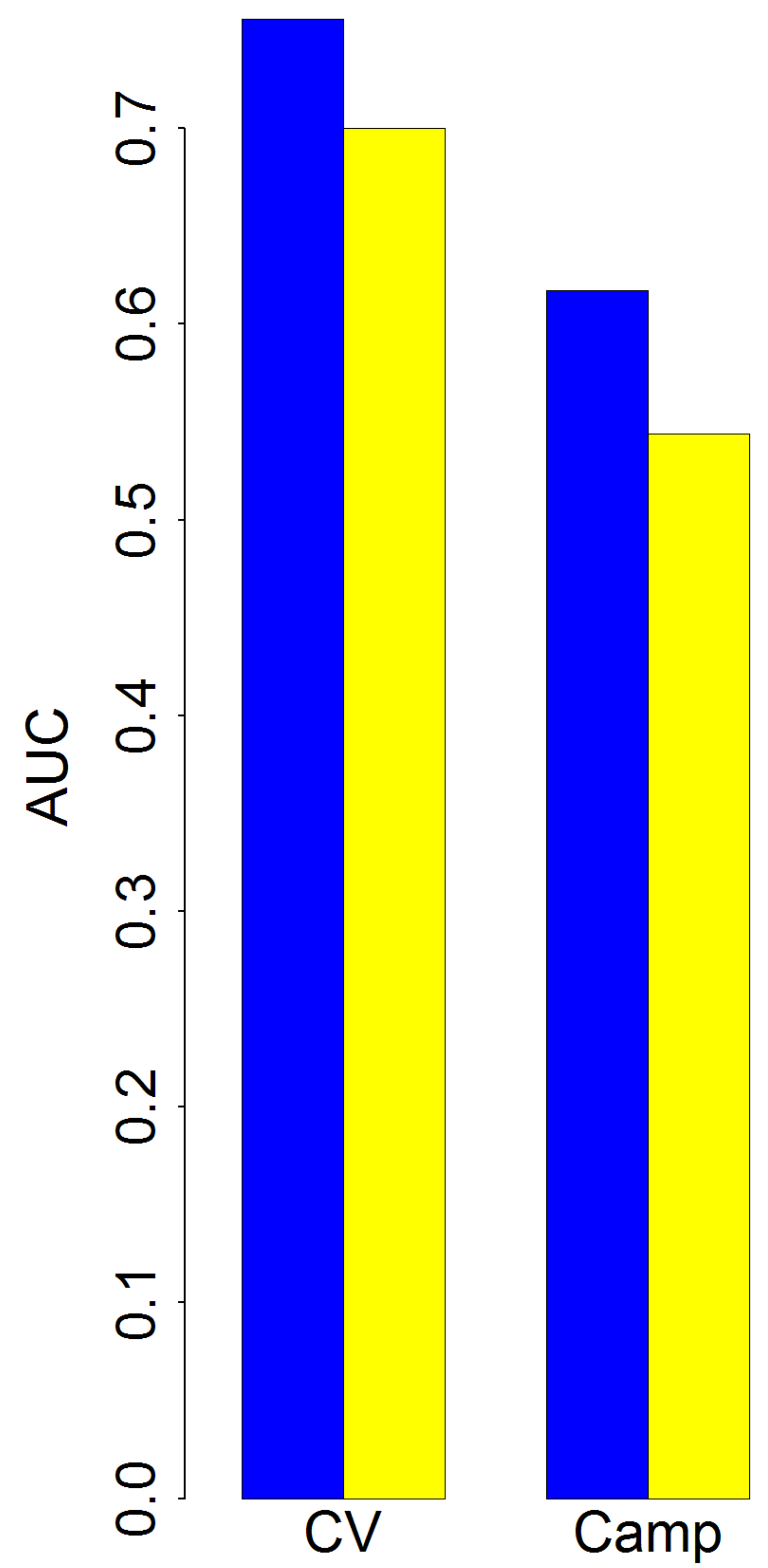

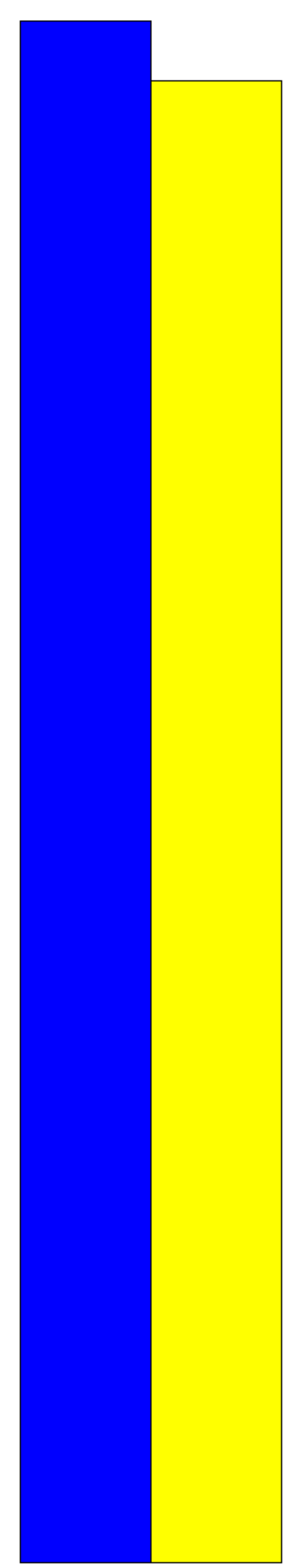

Epot

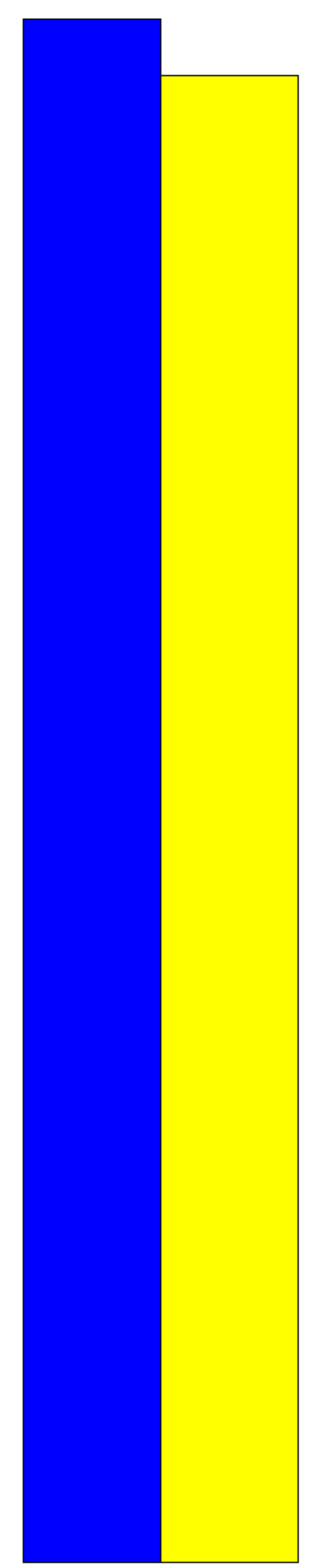

$\mathrm{Pacl}$

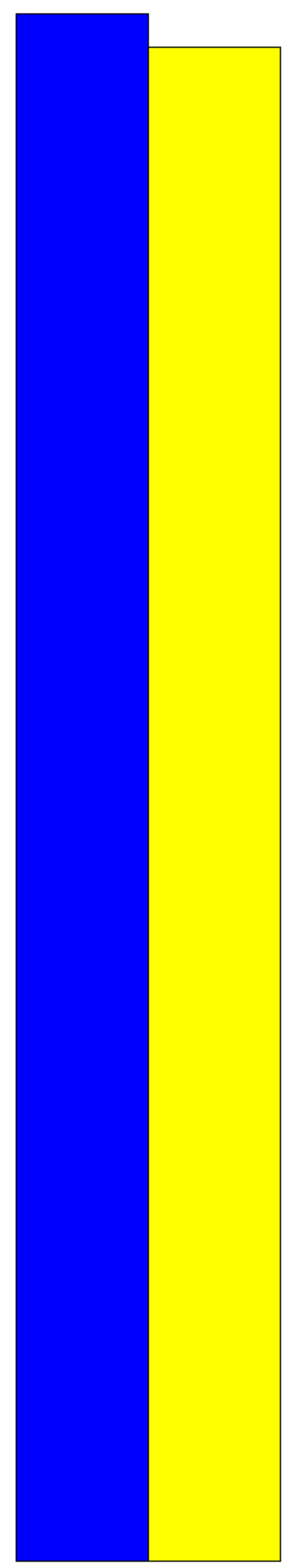

Shik

Top 50 features 


\section{Figure 3 (on next page)}

Comparison among different top $N$ significantly differential features .

AUCs plotted against different top $\mathrm{N}$ features in the case $\mathrm{K}=3$ are varied. J48, Decision Tree.

SVM, Support Vector Machines. RF, Random Forest. RoF, Rotation Forest. AUC, Area under the curve. 


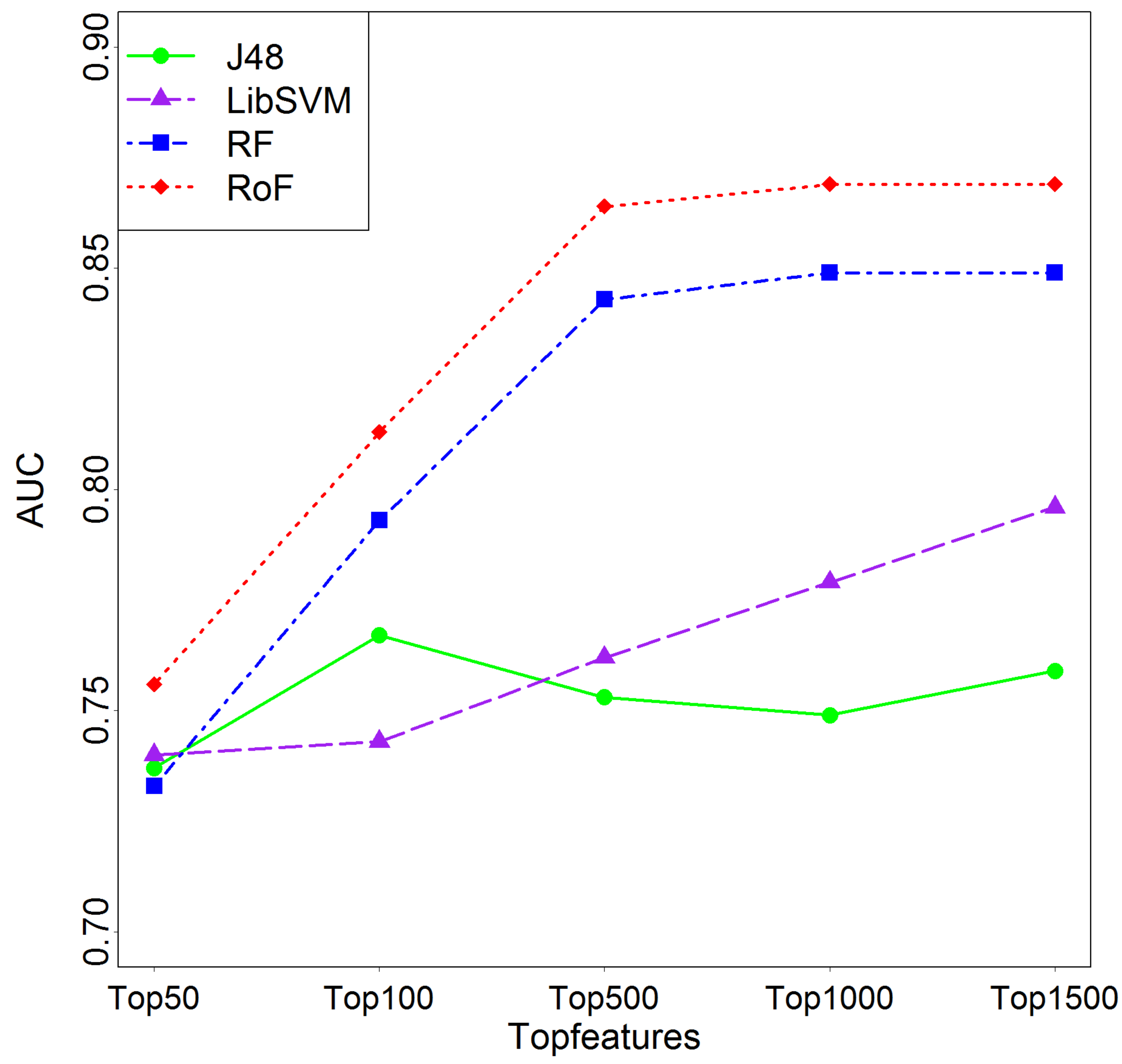


Figure 4 (on next page)

Comparison among different machine learning models .

ROC curve showing the proportion of true positives against the proportion of false positives as the classification threshold is varied for test set. (A), (B), (C) and (D) represent Camptothecin, Epothilone B, Paclitaxel and Shikonin, respectively. ROC, receiver operating characteristic. J48, Decision Tree. SVM, Support Vector Machines. RF, Random Forest. RoF, Rotation Forest. 

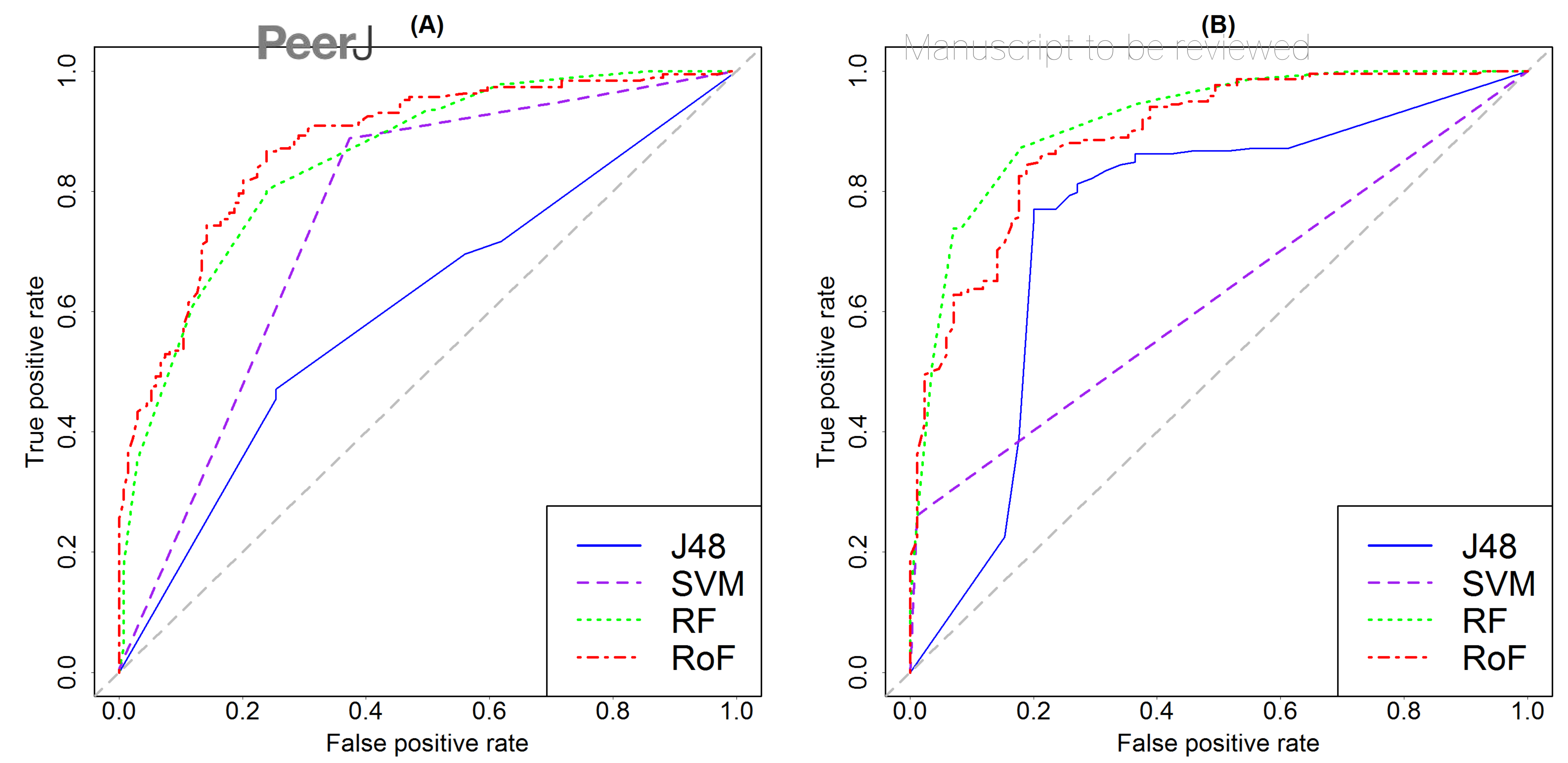

(C)
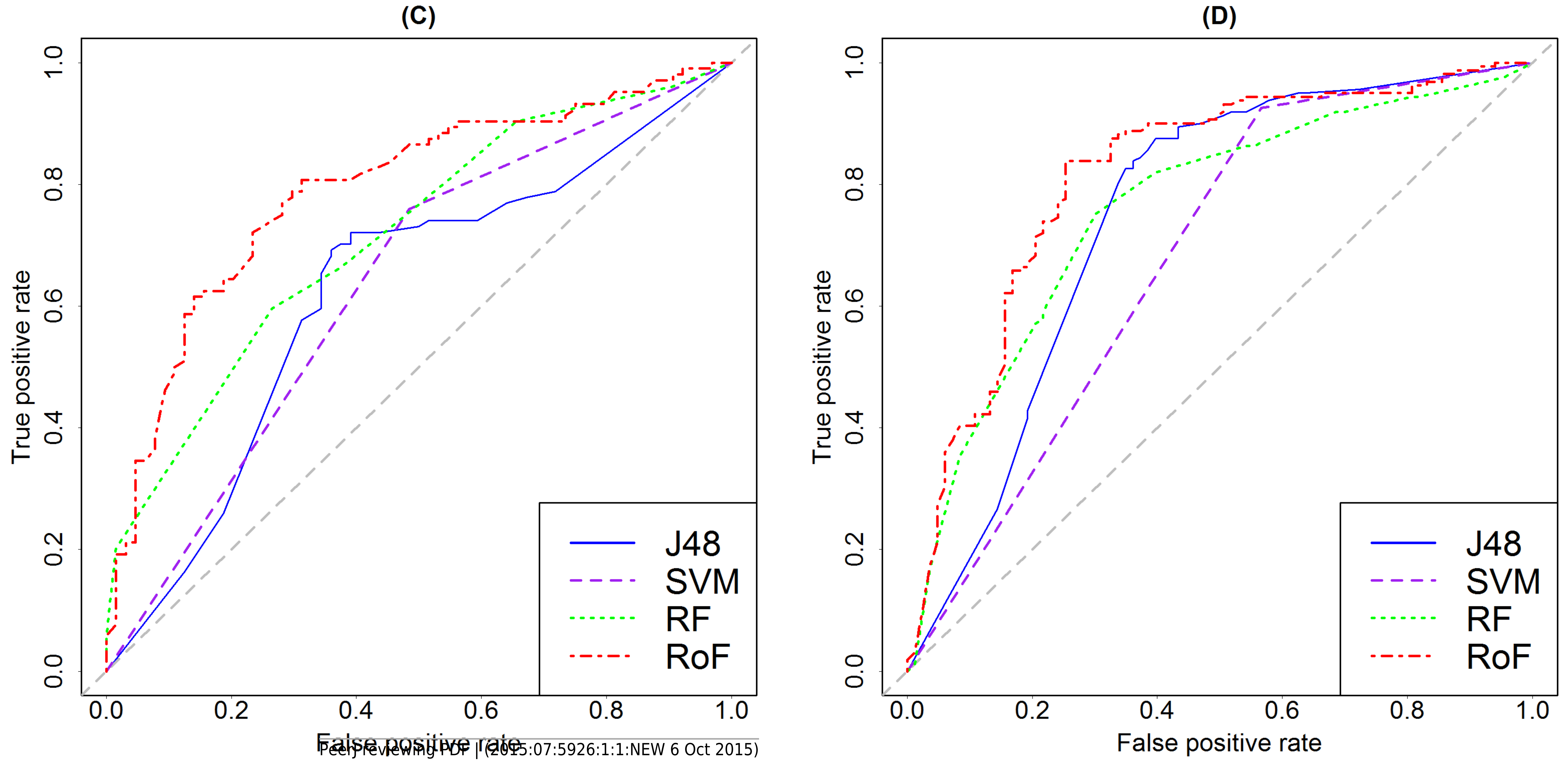